\title{
A Low-Cost Instrument for Estimating the Starch Content of Cassava Roots Based on the Measurement of RF Return Loss
}

\author{
Temitope Odedeyi*, Clive Poole*, Xinyue Liu*, Amany Kassem*, Gideon Oyebode ${ }^{\dagger}$, Rabbi Ismail ${ }^{\dagger}$, \\ and Izzat Darwazeh* \\ *Department of Electronic and Electrical Engineering, University College London (UCL), London, UK, WC1E 7JE \\ $\dagger$ International Institute of Tropical Agriculture (IITA), PMB 5320, Oyo Road, Ibadan 200001, Oyo State, Nigeria \\ \{temitope.odedeyi.13, c.poole, x.liu.17, amany.kassem.15, i.darwazeh\}@ucl.ac.uk, \{go.oyebode, i.rabbi\}@cgiar.org
}

\begin{abstract}
The problem of simply and reliably estimating starch content of cassava roots in the field is addressed by the development of a low cost test instrument that measures return loss at radio frequencies using a coaxial probe. A clear relationship between starch content of cassava roots and the measured return loss of root samples at a specific frequency of $30 \mathrm{MHz}$ is first verified experimentally. A prototype test instrument is then designed with goals of portability, low cost and simplicity of use. The test instrument displays starch content in 5 categories, from "low" to "high" using an array of 5 LEDs. The performance of the test instrument is experimentally verified in the field and a reliable correlation between cassava root starch content and LED indication is demonstrated.
\end{abstract}

Index Terms-Crop quality estimation, Cassava, Electrical impedance spectroscopy, Return Loss, Scalar Network Analysis, Test instrument design

\section{INTRODUCTION}

The characterisation of biological materials based on their interaction with electromagnetic energy in the Radio Frequency (RF) and microwave range, generally described as electrical impedance spectroscopy (EIS), dates back to 1894 [1]. The technique has generated significant interest in the food industry, where attempts have been made to identify different quality indices of crops and food substances based on their RF impedance properties [2] and a number of papers have been published on the use of EIS to characterise the physiological state of fruits [3]-[6]. EIS has also been applied to the characterisation of vegetable oils [6] and dairy products [7] and in measuring the concentration of pathogens in food samples such as milk, spinach and ground beef [8].

In the starch sub-industry, where new applications such as the production of bioenergy and biodegradable plastic alternatives are set to drive increasing global demand [9], the availability of a rapid, non-destructive method for estimating the starch content of fresh roots and tubers in the field is highly desirable [10]-[12]. Particular focus has been on the estimation of starch content in cassava (Manihot Esculenta), a tropical root vegetable which is one of the most important starch crops globally [13] and primary food staple in subSaharan Africa. The price paid for fresh cassava roots is often dependent on the estimated starch content [10]. The most accurate method of starch determination currently available is a qualitative laboratory analysis, which is beyond the reach of small and medium scale starch processors and most farmers as it requires specialised laboratory facilities [10]. A commonly adopted substitute is complete oven-drying, which leaves a dry-matter residue, $81.02 \%$ to $91.56 \%$ of which is pure starch
[14]. This Oven Dry Matter (ODM) technique is, however, mainly applicable in a research context as the process requires oven-drying at $105^{\circ} \mathrm{C}$ for at least 24 hours (much longer for lower oven temperature settings) to reach a steady dry weight [11], [12], [15]. A less accurate, but quicker and nondestructive, method of starch estimation is to determine the Root Specific Gravity (RSG) by measuring the weight of the roots in air and then in water [10]. The RSG is then used to estimate both dry-matter and starch content using empirical formulae such as those reported in [11]. The RSG method is the one most often used at starch production plants. Whilst being quicker and simpler to implement it can, however, be prone to significant measurement errors and requires well trained personnel to yield reliable results.

Studies have clearly suggested a link between the electrical properties, such as conductivity and dielectric constant, of fresh cassava root and its dry matter and starch content [16][18]. In this paper we describe work done to explore the link between starch content and the measured return loss of cassava root samples at a range of radio frequencies. This work resulted in the discovery of an optimum measurement frequency where difference in return loss between different starch content samples is most pronounced. We also describe the design and fabrication of a simple, low cost, hand-held test instrument that could potentially enable farmers to test starch content of cassava roots quickly and non-destructively in the field using the return loss method.

\section{EXPERIMENTAL INVESTIGATIONS}

A series of experiments were conducted at the International Institute of Tropical Agriculture (IITA) in Nigeria to determine the relationship between starch content and measured return loss across a range of radio frequencies up to $900 \mathrm{MHz}$. Three varieties of fresh cassava root, referred to as IITA-TMSIBA000070, IITA-TMS-IBA980505 and TMEB419, were obtained from the IITA research farms. Through the course of these experiments, over 250 roots were cut from the plants in the field and immediately transported to the laboratory, where they were cleaned and each cassava root divided into six sections: two sections each from the distal, mid and proximal regions. The extreme ends of the roots where cut off so that each section presented a viable test surface on both sides. One section each from the distal, mid and proximal regions were selected for return loss measurements, while the remaining portions were reserved for ODM processing. These sample portions were grated and homogenised by thorough mixing. 
$100 \mathrm{~g}$ portions of these gratings were then placed in the oven at $70^{\circ} \mathrm{C}$ for more than 48 hours to ensure complete drying. The dried samples were then weighed to determine percentage dry matter content. These ODM results were used to calibrate the return loss measurements against root sample starch content.

The return loss measurements were carried out using a PicoVNA-106 vector network analyzer and a purpose-made coaxial probe to measure the $\mathrm{S}$-parameter $S_{11}$ (equivalent to the one-port reflection coefficient), with the relationship between return loss in $\mathrm{dB}$ and the magnitude of $S_{11}$ being defined as [19]

$$
R L=-20 \log _{10}\left|S_{11}\right|
$$

An open-ended coaxial probe was used, since such probes have been the most commonly used method of determining the dielectric properties of semi-solid foods such as fresh fruits and vegetables [20]. This is also the most practical method for testing root crops in the field using a hand-held device. One consequence of taking spot measurements with a coaxial probe is that there will be marked variations in results depending on the location of the probe insertion point, and care needs to be taken to control for sample slice position and probe insertion point. The coaxial probe used in this work consisted of a semirigid solid copper-jacketed coaxial cable with one end stripped to expose a $3 \mathrm{~mm}$ section of the centre conductor. To ensure consistent depth of insertion, a band of insulating material was placed around the copper jacket at $5 \mathrm{~mm}$ from the tip of the probe.

Fig. 1 shows the complete test setup for the $S_{11}$ measurement, with an inset showing the oven drying setup. In all cases, the insertion point of the probe was the location of the central xylem bundle [21] in each slice, as shown in Fig. 1.

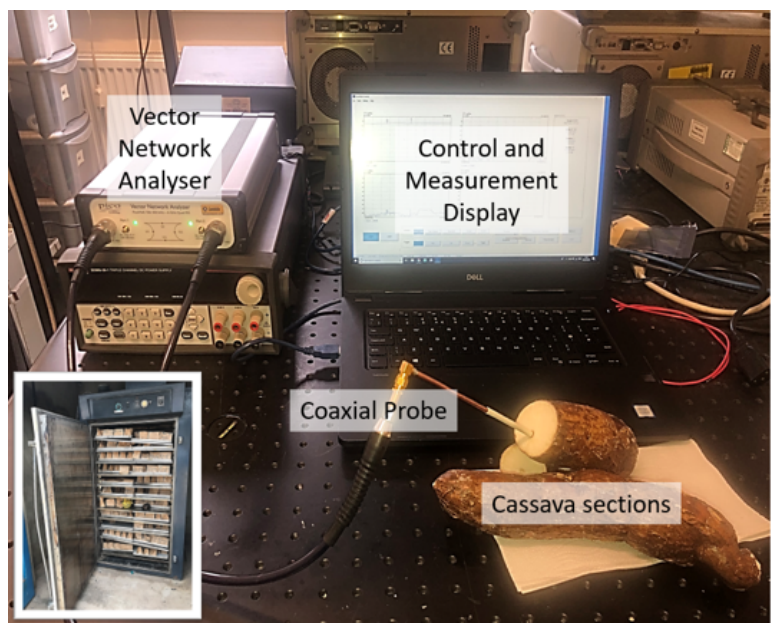

Fig. 1. Reflection measurements (S11) setup (Inset: Oven drying setup for dry matter and starch content estimation)

\section{DATA ANALYSis}

The plot of $\left|S_{11}\right|$ versus frequency for 46 cassava samples is shown in Fig. 2, with measurements taken from the distal portion. Each coloured trace represents an individual cassavasample. A general upward trend in the value of $\left|S_{11}\right|$ with frequency can be observed, with a pronounced minimum value of $\left|S_{11}\right|$ at around $30 \mathrm{MHz}$. This characteristic shape was observed for all sample sets, including those from the proximal and mid sections. The frequency of minimum $\left|S_{11}\right|$ is also the frequency at which the divergence in $\left|S_{11}\right|$ values for samples of differing starch content are most pronounced.

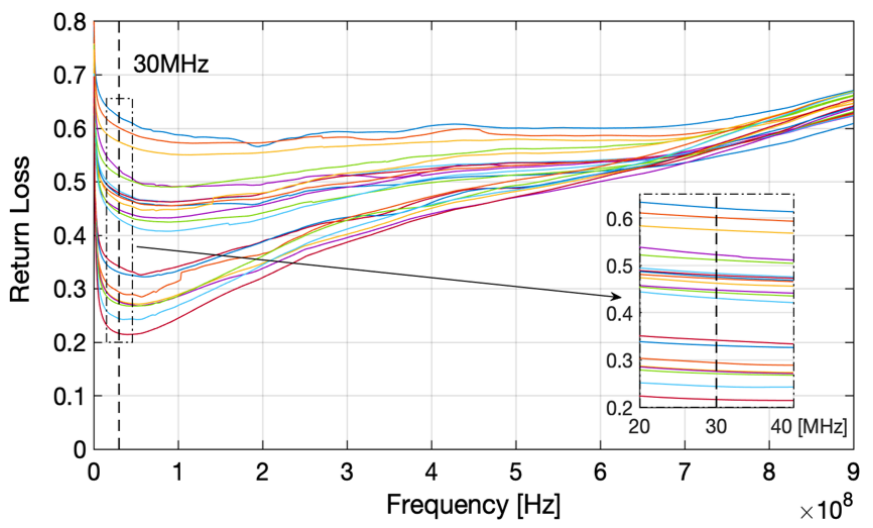

Fig. 2. S11 versus frequency for a typical cassava sample set.

Compared to the proximal and distal sections, $\left|S_{11}\right|$ measurements taken from the mid section of each cassava root tend to be lower, indicating a higher moisture content and proportionately lower dry matter content in the mid portion. This trend is clearly shown in Fig. 3, which compares $\left|S_{11}\right|$ measurements taken at $30 \mathrm{MHz}$, for the proximal, mid and distal sections of individual cassava roots. This is consistent with our current understanding of moisture and starch distribution within cassava roots [14].

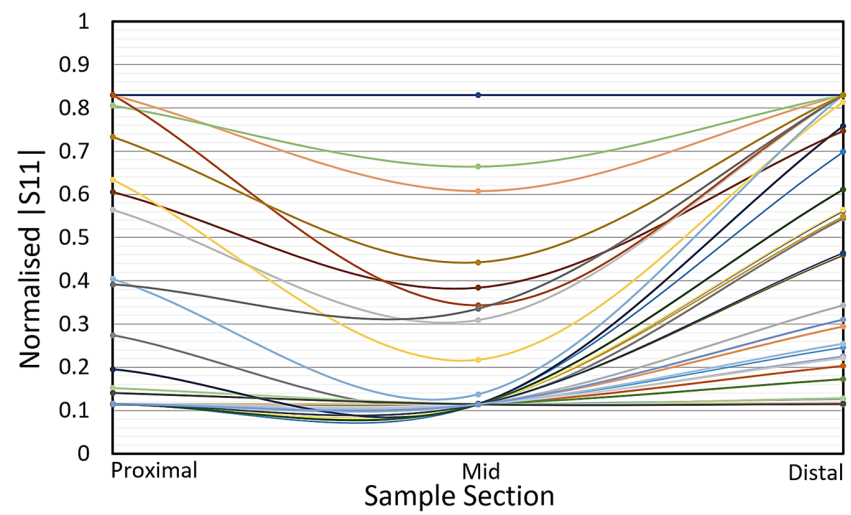

Fig. 3. Normalised S11 magnitude measured at $30 \mathrm{MHz}$ for proximal, mid and distal sections for a typical cassava samples.

A related phenomenon was observed for probe placement within the sample cross section, with measurements taken from the central xylem bundle tending to have a lower magnitude compared to the measurements taken towards the cortex of the sample slice. Taken together, these observations provide significant evidence that a spot frequency measurement of return loss at $30 \mathrm{MHz}$ is sufficient to classify cassava samples into "low", "medium" and "high" starch content, but that root slice selection and probe insertion point within the slice are important considerations in maintaining consistency of measurement results. 


\section{TEST INSTRUMENT DESIGN}

Having established RF return loss as a viable method of estimating the starch content of cassava roots, the goal was then to design a low cost, portable test instrument that could give an immediate, approximate indication of starch content in the field.

A simple test instrument was designed that generates a low power RF signal, at a single fixed frequency of $30 \mathrm{MHz}$, to be injected into the sample under test (SUT) via a coaxial probe. The test instrument then determines the amount of RF power reflected back from the sample via the probe. In this way the test instrument can be said to resemble a simple one-port scalar network analyser. The instrument calculates the return loss in $\mathrm{dB}$ as follows [19]

$$
R L_{S U T}=-20 \log _{10}\left(\left|\Gamma_{S U T}\right|\right)
$$

where $\Gamma_{S U T}$ is reflection coefficient of the SUT, defined by

$$
\Gamma_{S U T}=\frac{V^{-}}{V^{+}}
$$

where $V^{+}$is the incident voltage wave being injected into the SUT and $V^{-}$is the reflected voltage wave coming back from the SUT. The phase of the complex reflection coefficient seen at the input of the probe connector, $\Gamma_{x}$, will vary with the length of the probe, but the magnitude of $\Gamma_{x}$, and therefore the measured value of the return loss, is independent of probe length (assuming the probe is lossless).

In general a return loss of $20 \mathrm{~dB}$ or higher means that the percentage of the power reflected by the SUT is $1 \%$ or less of the incident power. On the other hand, if the return loss is between $0 \mathrm{~dB}$ and $3 \mathrm{~dB}$, then this means that the SUT is reflecting $50 \%$ or more of the incident power.

A block diagram of the test instrument is shown in fig. 4. It consists of a signal source, a receiver for detecting the signal, some simple data processing and an array of 5 LEDs to indicate a range of return loss values.

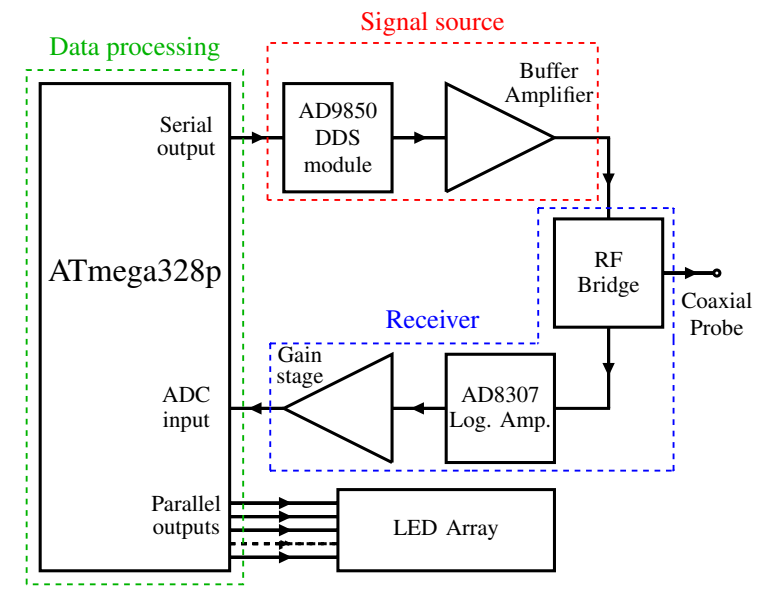

Fig. 4. Test instrument block diagram

The signal source employs the AD9850 Direct Digital Synthesizer (DDS) device from Analog Devices [22]. For simplicity, a DDS module containing the AD9850 device plus an external clock oscillator was used. The system controller is an ATMEL ATmega328p microcontroller in the form of an Arduino Nano module. At system power-up, the microcontroller provides a 32-bit frequency tuning word to the DDS module that sets the frequency of operation. The DDS module can generate any frequency in the range 0 and $40 \mathrm{MHz}$. In this case the frequency is set to $30 \mathrm{MHz}$, as this was established as the optimal test frequency in the previous experiments.

The output of the DDS is buffered by a single transistor common collector amplifier which prevents excessive loading of the DDS output by the widely varying impedances that will be presented by the SUT.

The receiver circuit employs an Analog Devices AD8307 logarithmic amplifier which produces an output voltage proportional to the common logarithm of the input signal amplitude, a response that is described as being "linear-in- $d B$ " [23]. A logarithmic amplifier was chosen over a simple diode detector in order to achieve wider dynamic range and higher measurement resolution. The AD8307 has a dynamic range of $92 \mathrm{~dB}$ with $\pm 3 \mathrm{~dB}$ law conformance at frequencies up to $100 \mathrm{MHz}$.

The analog inputs on the ATmega328p have 10-bit resolution with a full-scale voltage range of $5 \mathrm{~V}$. Since the output voltage of the AD8307 covers the range from $0.31 \mathrm{~V}$ (representing input RF power of $-73 \mathrm{dBm}$ ), to $2.45 \mathrm{~V}$ (representing input RF power of $10 \mathrm{dBm}$ ), an additional voltage gain stage is required between the AD8307 output and the analog input of the microcontroller in order to make use of the full dynamic range of the microcontroller ADC.

The buffered output from the DDS is injected into the SUT via the coaxial probe. When the probe is inserted into the sample it can be regarded as a transmission line with arbitrary load. The signal measured at the probe input is then the sum of both incident voltage wave injected into the line plus the reflected voltage wave being reflected back from the load. In order to measure return loss we need to isolate the reflected voltage wave from the incident voltage wave using an RF bridge [24], shown conceptually in Fig. 5 where the AD8307 takes the role of the RF detector.

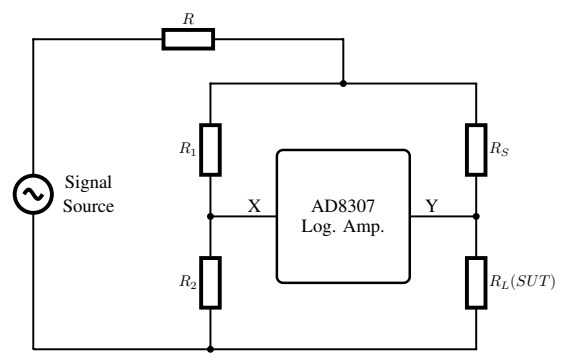

Fig. 5. RF Bridge concept

The voltage at point $Y$ is the vector sum of forward voltage $\left(V^{+}\right)$and reflected voltage $\left(V^{-}\right)$on the line, i.e. $V_{Y}=V^{+}+$ $V^{-}$. As we have set $R_{1}=R_{2}$, the voltage at point $X$ is simply a fraction of the forward voltage, $V^{+}$, as there is no reflected voltage component at $X$. Thus, when $R_{S}=R_{L}$, the 
RF detector reads $Y-X=V^{+}-V^{+}=0$. When there is a discontinuity at the tip of the probe, i.e. $R_{S} \neq R_{L}$, the bridge becomes unbalanced and the RF detector will read $Y-X=$ $V^{+}+V^{-}-V^{+}=V^{-}$. The signal presented to across AD8307 in such a bridge configuration will therefore represent only the reflected voltage wave coming back from the SUT.

A photograph of the completed prototype test instrument is shown in Fig. 6, which shows the DDS module (top right) the Arduino Nano (ATmega328p) module (centre) the probe SMA connector, RF bridge and AD8307 detector circuit (centre left) and the LED array (bottom right). The device consumes $610 \mathrm{~mW}$ DC power, and is powered using four AAA alkaline batteries.

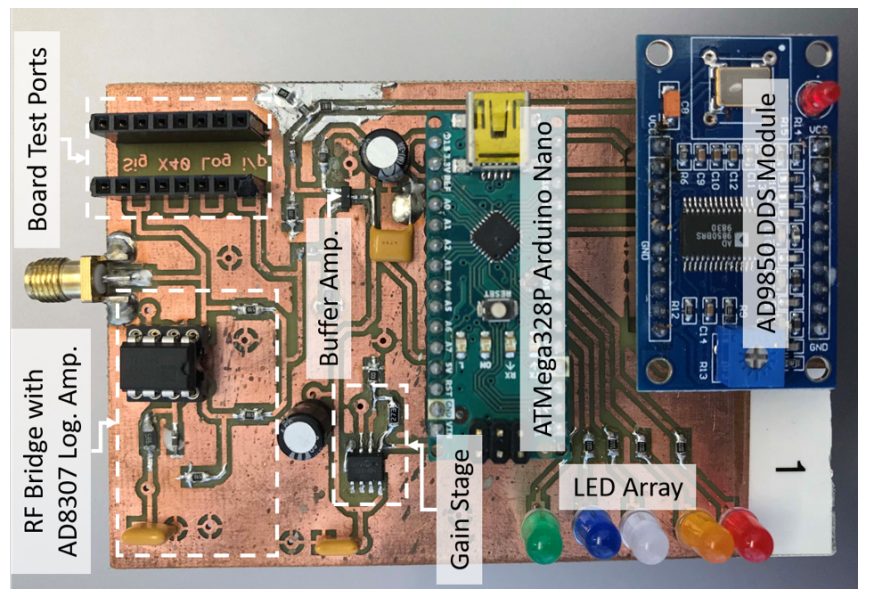

Fig. 6. Photograph of the prototype test instrument.

\section{TEST InSTRUMENT VALIDATION}

Four identical prototype test instruments were constructed and tested at IITA using 60 root samples taken from 4 different varieties of Cassava with starch content ranging between $6 \%$ and $22 \%$. The roots were cut into proximal, mid and distal sections and the starch content of each section was determined using the ODM method and the empirical formulae presented in [11]. The prototype test instrument was used to test each sample using a coaxial probe and the readings generated by the ATmega328p internal ADC, representing the measured return loss, were recorded. Fig. 7 shows average ADC readings against ODM measured starch content for the sample set together with the specific LED thresholds that were set during this test. A maximum ADC reading of 1024 represents a return loss of less than $3 \mathrm{~dB}$, i.e. more than half the incident RF power is being reflected back from the SUT. This corresponds to a high starch content and will be indicated by the green LED being activated. An ADC reading below 100 corresponds to a return loss of over $20 \mathrm{~dB}$ which will indicate that over $99 \%$ of incident RF power is being absorbed by the SUT. This corresponds to a low starch content and will be indicated by the red LED being activated.

Fig. 7 reveals a clear correlation between ADC output and ODM measured starch content, although the readings for mid slices were noticeably lower than those for proximal and distal slices, particularly at high starch levels. This is consistent with

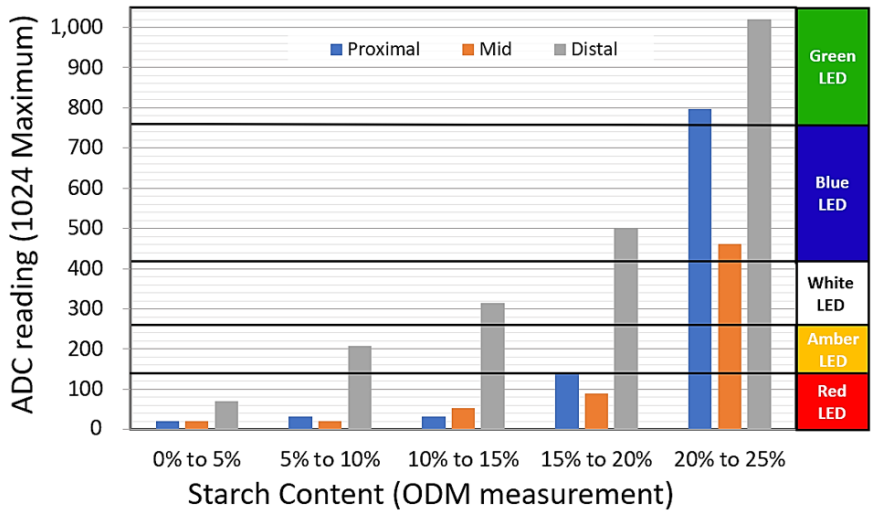

Fig. 7. Experimental results for the prototype test instrument, with LED thresholds set using measurement from distal sections.

our understanding of moisture distribution within the cassava root and our earlier results shown in Fig. 3. Fig. 7 would seem to suggest that the LED thresholds need to be set differently for different slice locations, but that the distal slice would appear to be the one giving the highest measurement resolution. Whilst starch content values above $25 \%$ are obtainable in high starch content cassava varieties at full maturation and peak season, such samples were not available to us during this project and the highest starch-content band used in our measurements was therefore $20 \%$ to $25 \%$. The test instrument is, however, capable of measuring much higher starch contents and the LED thresholds can be easily reset in software to allow for measurement of higher starch content varieties.

\section{CONCLUSiON}

A clear relationship between starch content of cassava roots and the measured return loss of root samples at a specific frequency of $30 \mathrm{MHz}$ has been demonstrated. A simple, low cost, test instrument was designed to allow the measurement of cassava root sample return loss in the field using a coaxial probe. For the purposes of simple classification, the test instrument displays starch content in 5 categories, from "low" to "high" using an array of 5 LEDs. The performance of the test instrument was experimentally verified and a reliable correlation between root starch content of between $0 \%$ and $25 \%$ and LED indication was demonstrated. The root slice position and location of probe insertion point within the slice were shown to be important considerations in obtaining consistent measurements. The low cost, portability and simplicity of use of the instrument makes it highly suitable for use by farmers in developing countries to determine the quality of cassava crops in the field.

\section{ACKNOWLEDGEMENT}

This work was funded by the UCL Global Challenges Research Fund under the Agri-tech for Sustainable Development: Crop Quality Evaluation and Prediction based on RF/Microwave Properties (ASPIRE) project. 


\section{REFERENCES}

[1] W. Nernst, "Methode zur bestimmung von dielektrizitätskonstanten," Zeitschrift für Physikalische Chemie, vol. 14, no. 1, pp. 622-663, 1894.

[2] M. Grossi and B. Riccò, "Electrical impedance spectroscopy (EIS) for biological analysis and food characterization: A review," 2017.

[3] F. R. Harker and J. H. Maindonald, "Ripening of nectarine fruit (changes in the cell wall, vacuole, and membranes detected using electrical impedance measurements)," Plant physiology, vol. 106, no. 1, pp. 165171, 1994.

[4] A. D. Bauchot, F. R. Harker, and W. M. Arnold, "The use of electrical impedance spectroscopy to assess the physiological condition of kiwifruit," Postharvest Biology and technology, vol. 18, no. 1, pp. 9-18, 2000.

[5] S. O. Nelson, "Dielectric spectroscopy of fresh fruits and vegetables," in 2005 IEEE Instrumentationand Measurement Technology Conference Proceedings, vol. 1. IEEE, 2005, pp. 360-364.

[6] H. Lizhi, K. Toyoda, and I. Ihara, "Dielectric properties of edible oils and fatty acids as a function of frequency, temperature, moisture and composition," Journal of Food Engineering, vol. 88, no. 2, pp. 151158, 2008.

[7] M. Mabrook and M. Petty, "Effect of composition on the electrical conductance of milk," Journal of food engineering, vol. 60, no. 3, pp. 321-325, 2003.

[8] L. Yang, Y. Li, and G. F. Erf, "Interdigitated array microelectrode-based electrochemical impedance immunosensor for detection of escherichia c oli o157: H7," Analytical chemistry, vol. 76, no. 4, pp. 1107-1113, 2004.

[9] A. Prakash, "Cassava: international market profile," Background paper for the competitive commercial agriculture in Sub-Saharan Africa (CCAA) study. Trade and Markets Division: Food and Agriculture Organisation (FAO), Rome, 2008.

[10] D. W. Wholey and R. H. Booth, "A comparison of simple methods for estimating starch content of cassava roots," Journal of the Science of Food and Agriculture, vol. 30, no. 2, pp. 158-164, 1979.

[11] E. Teye, A. Asare, R. Amoah, J. Tetteh et al., "Determination of the dry matter content of cassava (manihot esculenta, crantz) tubers using specific gravity method," ARPN Journal of Agricultural and Biological Science, vol. 6, no. 11, pp. 23-28, 2011.

[12] U. N. Ikeogu, F. Davrieux, D. Dufour, H. Ceballos, C. N. Egesi, and J.-L. Jannink, "Rapid analyses of dry matter content and carotenoids in fresh cassava roots using a portable visible and near infrared spectrometer (vis/nirs)," PloS one, vol. 12, no. 12, p. e0188918, 2017.

[13] N. K. Van Alfen, Encyclopedia of agriculture and food systems. Elsevier, 2014

[14] P. G. Figueiredo, M. A. d. Moraes-Dallaqua, S. J. Bicudo, and F. Y. Tanamati, "Starch accumulation in cassava roots: Spatial and temporal distribution," African Journal of Agricultural Research, pp. 5712-5712, 2013.

[15] N. U. Haase, "Estimation of dry matter and starch concentration in potatoes by determination of under-water weight and near infrared spectroscopy," Potato Research, vol. 46, no. 3, pp. 117-127, Sep 2003. [Online]. Available: https://doi.org/10.1007/BF02736081

[16] S. Harnsoongnoen and A. Siritaratiwat, "Electrical and absorption properties of fresh cassava tubers and cassava starch," Journal of Instrumentation, vol. 10, pp. P09 004-P09 004, 092015.

[17] P. Bergo, I. C. F. Moraes, and P. J. d. A. Sobral, "Effects of moisture content on structural and dielectric properties of cassava starch films," Starch-Stärke, vol. 64, no. 10, pp. 835-839, 2012.

[18] O. O. Ajibola, "Moisture dependence of some electrical and dielectric properties of cassava," Journal of the Science of Food and Agriculture, vol. 36, no. 5, pp. 359-366, 1985.

[19] C. Poole and I. Darwazeh, Microwave Active Circuit Analysis and Design. Elsevier Science, 2015.

[20] S. N. Jha, K. Narsaiah, A. Basediya, R. Sharma, P. Jaiswal, R. Kumar, and R. Bhardwaj, "Measurement techniques and application of electrical properties for nondestructive quality evaluation of foods-a review," Journal of food science and technology, vol. 48, no. 4, pp. 387-411, 2011.

[21] I. Onwueme, The tropical tuber crops: yams, cassava, sweet potato, and cocoyams. John Wiley and Sons, 1978.

[22] Analog Devices, "CMOS, $125 \mathrm{MHz}$ Complete DDS Synthesizer," AD9850 Analog Devices Inc, 1999.
[23] Matthew Pilotte, "Operation of RF Detector Products at Low Frequency," Application Note AN-691, Analog Devices Inc., 2005.

[24] P. C. L. Yip, High-Frequency Circuit Design and Measurements. Chapman and Hall, 1990. 